\title{
Correlation Analysis Between Preoperative Systemic Immune Inflammation Index and Prognosis of Gastric Carcinoma Patients: Based on Propensity Score Matching Method
}

\author{
Zhaojun Xu \\ Qinghai University https://orcid.org/0000-0001-9275-9652 \\ Xiaobin Chen \\ Qinghai University \\ Juan An \\ Qinghai University \\ Jiaqi Yuan \\ Qinghai University \\ Shuyun Jiang \\ Qinghai University \\ Tao Liu \\ Qinghai University \\ Baojia Cai \\ Qinghai University \\ Cheng Wang \\ Qinghai University \\ Xiaoming Ma ( $\sim$ hilton007@126.com )
}

Qinghai University Affiliated Hospital https://orcid.org/0000-0003-4539-7438

\section{Research}

Keywords: gastric carcinoma, systemic immune inflammation index, prognosis, overall survival, propensity score matching method

Posted Date: July 26th, 2021

DOI: https://doi.org/10.21203/rs.3.rs-725912/v1

License: (9) (i) This work is licensed under a Creative Commons Attribution 4.0 International License. Read Full License 


\section{Abstract}

Background. To explore the correlation between the preoperative systemic immune inflammation index (SII) and the prognosis of patients with gastric carcinoma (GC).

Methods: The clinical data of 771 GC patients surgically treated in the Department of Gastrointestinal Surgery, Qinghai University Affiliated Hospital from June 2010 to June 2015 were retrospectively analyzed, and their preoperative SII was calculated. The optimal cut-off value of preoperative SIl was determined using the receiver operating characteristic (ROC) curve, the confounding factors between the two groups were eliminated using the propensity score matching (PSM) method, and the correlation between preoperative SII and clinicopathological characteristics was assessed by chi-square test. Moreover, the overall survival was calculated using Kaplan-Meier method, the survival curve was plotted, and log-rank test was performed for the significance analysis between the curves. Univariate and multivariate analyses were also conducted using the Cox proportional hazards model.

Results. It was determined by the ROC curve that the optimal cut-off value of preoperative SII was 489.52, based on which $771 \mathrm{GC}$ patients were divided into high SII (H-SII) group and low SII (L-SII) group, followed by PSM in the two groups. The results of Kaplan-Meier analysis showed that before and after PSM, the postoperative 1-, 3- and 5-year survival rates in L-SII group were superior to those in H-SIl group, and the overall survival rate had a statistically significant difference between the two groups $(P<0.05)$. Before PSM, preoperative SII [hazard ratio $(H R)=2.707,95 \%$ confidence interval $(\mathrm{Cl}): 2.074-3.533, P<0.001$ ] was an independent risk factor for the prognosis of GC patients. After 1:1 PSM, preoperative SII (HR=2.669, 95\%Cl: 1.881-3.788, $\mathrm{P}<0.001)$ was still an independent risk factor for the prognosis of GC patients.

Conclusions. Preoperative SII is an independent risk factor for the prognosis of GC patients. The increase in preoperative SII in peripheral blood indicates a worse prognosis.

\section{Introduction}

Gastric carcinoma (GC) is a common digestive tract malignancy. In 2018, there were up to 782,000 deaths of GC, making it the third cause of death in malignancies ${ }^{[1]}$. China is a GC-prone country, and the new cases and deaths account for $44.1 \%$ and $49.9 \%$, respectively, of the total globally, with an age-standardized 5-year survival rate of $27.4 \%$. At the same time, GC patients have insidious early symptoms and the therapeutic effect is poor, leading to a poor prognosis ${ }^{\text {[2] }}$. Therefore, researchers have been constantly exploring the new simple, economical and accurate prognostic evaluation index similar to TNM stage currently, so as to better guide the clinical treatment ${ }^{[3]}$.

According to related studies, both inflammatory response and immune response are closely related to the occurrence and development of tumors ${ }^{[4,5]}$. Tumor-induced inflammatory response can cause corresponding changes in the blood neutrophil $(\mathrm{NE})$, lymphocyte $(\mathrm{LY})$ and platelet $(\mathrm{PLT})$ counts ${ }^{[6,7,8]}$. On this basis, some studies have tried to discover new potential biomarkers related to the prognosis. For example, the preoperative systemic immune inflammation index (SII) calculated based on NE, LY and PLT is correlated with the prognosis of breast cancer, liver cancer and pancreatic cancer ${ }^{[9,10,11,12]}$. However, there are currently few studies on the correlation between SII and prognosis of GC. In the present study, therefore, the value of SII for the prognosis of GC patients was explored using propensity score matching (PSM), so as to provide references for clinical treatment.

\section{Methods}

This study was agreed by the Institutional Research Ethics Board of Qinghai University Affiliated Hospital and obtain the informed consent of all subjects themselves or their families. All methods were performed in accordance with the Declaration of Helsinki, and this study did not involve human or animal tissue or blood samples, and all patients signed the written informed consent before surgery.

\section{General data}


The clinical data of GC patients surgically treated in the Department of Gastrointestinal Surgery, Qinghai University Affiliated Hospital from June 2010 to June 2015 were retrospectively analyzed. The treatment methods included distal gastrectomy (DG), proximal gastrectomy (PG) and total gastrectomy (TG). Inclusion criteria were as follows: (1) patients pathologically diagnosed with gastric mucosal adenocarcinoma after operation, (2) those without undergoing radiotherapy, chemotherapy and biotherapy before operation, (3) those without acute/chronic inflammation before operation, (4) those undergoing standard D2 gastrectomy, (5) those without other severe concomitant diseases and with good organ function, and (6) those without medical contraindications that seriously affect anesthesia and operation. Exclusion criteria were as follows: (1) patients with missing medical data or lost to follow-up, (2) those who refused to undergo operation, (3) those complicated with blood system diseases or other tumors before operation, (4) those with severe uncontrolled recurrent infections, or other severe uncontrolled concomitant diseases, or (5) those who require immunosuppressive therapy due to organ transplantation. The pathological staging was based on the $8^{\text {th }}$ edition UICC/AJCC staging criteria ${ }^{[13]}$, and the pathological diagnosis and classification of GC were based on the Japanese Gastric Cancer Treatment Guidelines (5 ${ }^{\text {th }}$ edition) ${ }^{[14]}$.

\section{Analysis methods}

SII was calculated using the blood routine test results first time after admission (SII = PLT $\times$ NE/LY). According to the optimal cutoff value of SII, the patients were grouped, and the correlation between SII and clinicopathological factors of patients was analyzed. The patients were also divided into non-elderly group ( $<60$ years old) and elderly group ( $\geq 60$ years old). The correlation between SII and patients' age and its influence on prognosis were analyzed.

\section{PSM}

This was a retrospective study, the data were not strictly randomized and there were many potential influencing factors for SII in clinic, so various confounding factors might be distributed in GC patients with different SII. To eliminate the influence of confounding factors on the prognosis, 1:1 PSM was performed for patients in high SII (H-SII) group and low SII (L-SII) group. Other covariates had no statistically significant difference $(P>0.05)$.

\section{Follow-up}

All patients were regularly followed up after operation every 3-6 months by outpatient clinic, message, telephone, e-mail and network communication tools. Overall survival (OS) was defined as the duration from definite diagnosis to death or end of followup (February 2021 or patients' death).

\section{Statistical analysis}

SPSS 26.0, R 3.6.1 and GraphPad Prism 8.0 were used for statistical analysis. Enumeration data were expressed as n (\%), and $\chi^{2}$ test was performed for intergroup comparison. The optimal cut-off value of SIl was determined using the receiver operating characteristic (ROC) curve. The confounding factors in data were eliminated using PSM, and the matching precision was set to 0.02. OS was calculated using Kaplan-Meier method, the survival curve was plotted, and log-rank test was performed for the difference between groups. Univariate and multivariate analyses were also conducted using the Cox proportional hazards model. The hazard ratio (HR) and the corresponding $95 \%$ confidence interval $(95 \% \mathrm{Cl})$ were calculated. $\mathrm{P}<0.05$ was considered to be statistically significant.

\section{Results}

\section{General data}

A total of 771 patients met the screening criteria, including 165 females $(21.40 \%)$ and 606 males (78.60\%), with a median age of 59.30 years old (Fig. 1). All patients were pathologically diagnosed with gastric mucosal adenocarcinoma after operation, and none of them died of postoperative complications. Among all patients, 297 cases (38.52\%) underwent DG, 250 cases (32.43\%) underwent PG, and 224 cases (29.05\%) underwent TG. Standard D2 lymph node dissection was conducted for all patients. All of the 771 patients were followed up until February 6, 2021, with a median follow-up time of 46 months (95\%Cl: 43.986-48.014). 


\section{Optimal Cut-off Value Of Sii}

The ROC curve of SIl was plotted, and its AUC and Youden index were 0.721 (95\% Cl: $0.681-0.761)$ and 0.384 , respectively. The corresponding optimal cut-off value was 489.52 , and the evaluation sensitivity and specificity were $55.5 \%$ and $82.9 \%$, respectively (Fig. 2). Based on the cut-off value, 771 patients were divided into L-SII group (SII $\leq 489.52, \mathrm{n}=531$ ) and $\mathrm{H}$-SII group (SII > 489.52, $n=240)$.

Correlation between SII and clinicopathological factors of GC patients before and after PSM

Before matching, SIl was related to the maximum diameter of tumor, histological type, serum albumin (ALb), white blood cell (WBC), carbohydrate antigen 125 (CA125) and TNM stage $(P<0.05)$, but not related to the age, gender, smoking, drinking, carcinoembryonic antigen (CEA), CA199 and operation method $(P>0.05)$. PSM was performed in L-SIl group and H-SIl group, and a total of 354 patients were eligible, including 177 cases in H-SIl group and 177 cases in L-SIl group. The above differences were evenly distributed after matching $(P>0.05)$ (Table 1). 
Correlation between SII and clinicopathological factors of GC patients before and after PSM

\begin{tabular}{|c|c|c|c|c|c|c|c|c|}
\hline \multirow[t]{2}{*}{ Parameters } & \multicolumn{4}{|l|}{ Before PSM } & \multicolumn{4}{|l|}{ After PSM } \\
\hline & $\begin{array}{l}\text { H-SII }(n= \\
240)\end{array}$ & $\underset{531)}{\text { L-SII }(n=}$ & $x^{2}$ & $\mathbf{P}$ & $\begin{array}{l}\text { H-SII }(n= \\
177)\end{array}$ & $\begin{array}{l}\text { L-SII }(n= \\
177)\end{array}$ & $x^{2}$ & $\mathbf{P}$ \\
\hline Age(years) & & & 3.415 & 0.065 & & & 0.011 & 0.915 \\
\hline$\leq 60$ & $129(53.75 \%)$ & $323(60.83 \%)$ & & & $100(56.50 \%)$ & $99(55.93 \%)$ & & \\
\hline$>60$ & $111(46.25 \%)$ & 208(39.17\%) & & & $77(43.50 \%)$ & $78(44.07 \%)$ & & \\
\hline Gender & & & 0.097 & 0.756 & & & 0.000 & 1.000 \\
\hline Male & 187(77.92\%) & $419(78.91 \%)$ & & & $138(77.97 \%)$ & $138(77.97 \%)$ & & \\
\hline Female & $53(22.08 \%)$ & 112(21.09\%) & & & $39(22.03 \%)$ & $39(22.03 \%)$ & & \\
\hline Smoking & & & 0.234 & 0.628 & & & 0.011 & 0.915 \\
\hline Yes & $113(47.08 \%)$ & $260(48.96 \%)$ & & & $84(47.46 \%)$ & $83(46.89 \%)$ & & \\
\hline No & $127(52.92 \%)$ & $271(51.04 \%)$ & & & $93(52.54 \%)$ & $94(53.11 \%)$ & & \\
\hline Drinking & & & 1.246 & 0.264 & & & 1.457 & 0.227 \\
\hline Yes & $91(37.92 \%)$ & $224(42.18 \%)$ & & & $72(40.68 \%)$ & $61(34.46 \%)$ & & \\
\hline No & $149(62.08 \%)$ & $307(57.82 \%)$ & & & $105(59.32 \%)$ & $116(65.54 \%)$ & & \\
\hline Tumor size (mm) & & & 11.357 & $\begin{array}{l}< \\
0.001\end{array}$ & & & 0.232 & 0.630 \\
\hline$\leq 50$ & $168(70.00 \%)$ & $429(80.79 \%)$ & & & $128(72.32 \%)$ & $132(74.58 \%)$ & & \\
\hline$>50$ & $72(30.00 \%)$ & 102(19.21\%) & & & $49(27.68 \%)$ & $45(25.42 \%)$ & & \\
\hline Differentiation & & & 4.188 & 0.041 & & & 0.000 & 1.000 \\
\hline Well and Moderately & $73(30.42 \%)$ & 202(38.04\%) & & & $61(34.46 \%)$ & $61(34.46 \%)$ & & \\
\hline Poorly & $167(69.58 \%)$ & $329(61.96 \%)$ & & & $116(65.54 \%)$ & $116(65.54 \%)$ & & \\
\hline Alb (g/L) & & & 33.695 & $\begin{array}{l}< \\
0.001\end{array}$ & & & 0.000 & 1.000 \\
\hline$\leq 39.85$ & $183(76.25 \%)$ & $288(54.24 \%)$ & & & $121(68.36 \%)$ & $121(68.36 \%)$ & & \\
\hline$>39.85$ & $57(23.75 \%)$ & $243(45.76 \%)$ & & & $56(31.64 \%)$ & $56(31.64 \%)$ & & \\
\hline WBC $(W, \times 109$ cells $/ L)$ & & & 107.173 & $\begin{array}{l}< \\
0.001\end{array}$ & & & 0.113 & 0.737 \\
\hline$\leq 6.525$ & $119(49.58 \%)$ & $451(84.93 \%)$ & & & $118(66.67 \%)$ & $115(64.97 \%)$ & & \\
\hline$>6.525$ & $121(50.41 \%)$ & $80(15.07 \%)$ & & & $59(33.33 \%)$ & $62(35.03 \%)$ & & \\
\hline CEA & & & 0.542 & 0.462 & & & 0.554 & 0.457 \\
\hline$\leq 2.17 \mathrm{ug} / \mathrm{ml}$ & $128(53.33 \%)$ & $268(50.47 \%)$ & & & $89(50.28 \%)$ & $82(46.33 \%)$ & & \\
\hline$>2.17 \mathrm{ug} / \mathrm{ml}$ & $112(46.67 \%)$ & $263(49.53 \%)$ & & & $88(49.72 \%)$ & $95(53.67 \%)$ & & \\
\hline CA199 & & & 0.835 & 0.361 & & & 0.0137 & 0.711 \\
\hline$\leq 21 \mathrm{u} / \mathrm{mL}$ & $179(74.58 \%)$ & $412(77.59 \%)$ & & & 135(76.27\%) & $132(74.58 \%)$ & & \\
\hline$>21 \mathrm{u} / \mathrm{mL}$ & $61(25.42 \%)$ & $119(22.41 \%)$ & & & $42(23.73 \%)$ & $45(25.42 \%)$ & & \\
\hline
\end{tabular}




\begin{tabular}{|c|c|c|c|c|c|c|c|c|}
\hline \multirow[t]{2}{*}{ Parameters } & \multicolumn{4}{|l|}{ Before PSM } & \multicolumn{4}{|l|}{ After PSM } \\
\hline & $\begin{array}{l}\text { H-SII }(n= \\
240)\end{array}$ & $\begin{array}{l}\text { L-SII }(n= \\
531)\end{array}$ & $x^{2}$ & $\mathbf{P}$ & $\begin{array}{l}\text { H-SII }(n= \\
177)\end{array}$ & $\begin{array}{l}\text { L-SII }(n= \\
177)\end{array}$ & $\chi^{2}$ & $\mathbf{P}$ \\
\hline CA125 & & & 6.671 & 0.010 & & & 1.532 & 0.216 \\
\hline$\leq 14.6 \mathrm{u} / \mathrm{ml}$ & $145(60.42 \%)$ & $371(69.87 \%)$ & & & 112(63.28\%) & $123(69.49 \%)$ & & \\
\hline$>14.6 \mathrm{u} / \mathrm{ml}$ & $95(39.58 \%)$ & $160(30.13 \%)$ & & & $65(36.72 \%)$ & $54(30.51 \%)$ & & \\
\hline Type of surgery & & & 5.572 & 0.062 & & & 4.974 & 0.084 \\
\hline DG & $80(33.33 \%)$ & $217(40.87 \%)$ & & & $57(32.21 \%)$ & $39(22.04 \%)$ & & \\
\hline PG & $78(32.50 \%)$ & $172(32.39 \%)$ & & & $59(33.33 \%)$ & $63(35.59 \%)$ & & \\
\hline TG & $82(34.17 \%)$ & $142(26.74 \%)$ & & & $61(34.46 \%)$ & $75(42.37 \%)$ & & \\
\hline TNM stage & & & 11.509 & 0.003 & & & 2.425 & 0.298 \\
\hline I & $38(15.83 \%)$ & $137(25.80 \%)$ & & & $34(16.95 \%)$ & $35(19.77 \%)$ & & \\
\hline ॥ & $43(17.92 \%)$ & 105(19.78\%) & & & $31(17.51 \%)$ & $40(22.60 \%)$ & & \\
\hline III & $159(66.25 \%)$ & $289(54.42 \%)$ & & & $116(65.54 \%)$ & $102(57.63 \%)$ & & \\
\hline
\end{tabular}

Correlation between SII and OS of GC patients before and after PSM

The median survival time was 73 months (95\% Cl: 65.172-80.828) in L-SIl group and 28 months (95\%Cl: $24.646-31.354)$ in $\mathrm{H}-\mathrm{SII}$ group. The 1-, 3- and 5-year survival rates were $92.8 \%, 81.0 \%$ and $67.9 \%$, respectively, in L-SIl group, and $80.0 \%, 39.8 \%$ and $28.9 \%$, respectively, in H-SII group. It can be seen that the survival rate in L-SII group was superior to that in H-SII group, and the OS rate had a statistically significant difference between the two groups $(\mathrm{P}<0.001)$. After PSM, the 1-, 3- and 5-year survival rates were $89.3 \%, 74.3 \%$ and $60.4 \%$, respectively, in L-SII group, and $80.8 \%, 45.8 \%$ and $32.9 \%$, respectively, in H-SIl group. The mean survival time was 56.771 months (95\% Cl: $51.613-61.929)$ in L-SIl group. In H-SIl group, the mean survival time was 38.443 months (95\%Cl: $34.792-42.093)$, and the median survival time was 32 months ( $95 \% \mathrm{Cl}$ : $22.446-41.554)$. It can be seen that the survival rate in L-SII group was significantly better than that in H-SII group, and the OS rate had a statistically significant difference between the two groups $(P<0.001)$ (Fig. 3).

\section{Influencing Factors For Patient'S Survival Before And After Psm}

Before PSM, the results of univariate analysis revealed that age, maximum diameter of tumor, histological type, SII, ALb, CEA, CA199, CA125, WBC count, operation method and TNM stage were all influencing factors for the prognosis of GC patients ( $<<$ 0.05). The results of multivariate analysis showed that age $(\mathrm{HR}=1.334,95 \% \mathrm{Cl}: 1.045-1.704, \mathrm{P}=0.021)$, histological type $(\mathrm{HR}=$ $0.741,95 \% \mathrm{Cl}: 0.560-0.980, \mathrm{P}=0.036)$, SII (HR = 2.707, 95\%Cl: 2.074-3.533, $\mathrm{P}<0.001)$, ALb (HR = 0.385, 95\%Cl: 0.283-0.525, $\mathrm{P}<$ 0.001), CEA (HR=1.285, 95\%Cl: 1.007-1.640, $P=0.044), C A 125(H R=1.370,95 \% C l: 1.069-1.755, P=0.013), W B C(H R=1.361$, 95\%Cl: 1.047-1.770, $\mathrm{P}=0.021)$, operation method and TNM stage were all independent risk factors for the prognosis of GC patients (Table 2). After PSM, the results of univariate analysis manifested that age, maximum diameter of tumor, SII, ALb, CA125, operation method and TNM stage were risk factors for the prognosis of GC patients. According to multivariate analysis, the maximum diameter of tumor $(\mathrm{HR}=1.493,95 \% \mathrm{Cl}: 1.043-2.136, \mathrm{P}=0.029)$, SII $(\mathrm{HR}=2.669,95 \% \mathrm{Cl}: 1.881-3.788, \mathrm{P}<0.001)$, ALb (HR = 0.286, 95\%Cl: 0.183-0.447, P< 0.001), CA125 (HR=1.402, 95\%Cl: 1.002-1.961, P=0.048), operation method and TNM stage were independent risk factors for the prognosis of GC patients (Table 3). Besides, the correlation between statistically significant independent risk factors after PSM $(\mathrm{P}<0.05)$ (except SII) and prognosis of GC patients was analyzed by Kaplan-Meier method. It was found that the larger maximum diameter of tumor, lower level of ALb, higher level of CA125 and higher TNM stage 
indicated a worse prognosis. Besides, the prognosis was better among patients undergoing DG than that among patients treated with PG and TG (Fig. 4).

Table 2

Risk factors for the prognosis of GC patients before PSM

\begin{tabular}{|c|c|c|c|c|}
\hline \multirow[t]{2}{*}{ Parameters } & \multicolumn{2}{|l|}{ Univariate analysis } & \multicolumn{2}{|l|}{ Multutivariate analysis } \\
\hline & $\mathrm{HR}(95 \% \mathrm{Cl})$ & $\mathbf{P}$ & $\mathrm{HR}(95 \% \mathrm{Cl})$ & $\mathbf{P}$ \\
\hline $\operatorname{Age,y}(\leq 60$ VS. $>60)$ & $1.531(1.209-1.938)$ & $<0.001$ & $1.334(1.045-1.704)$ & 0.021 \\
\hline Gender(male VS. female) & $0.792(0.584-1.072)$ & 0.131 & & \\
\hline Smoking(yes VS. no) & $0.913(0.722-1.155)$ & 0.448 & & \\
\hline Drinking(yes VS. no) & $0.981(0.774-1.244)$ & 0.876 & & \\
\hline Tumor size,mm( $\leq 5$ VS. >5) & $1.619(1.253-2.091)$ & $<0.001$ & $1.182(0.903-1.547)$ & 0.223 \\
\hline Differentiation(Poorly VS. Well and Moderately ) & $0.605(0.462-0.794)$ & $<0.001$ & $0.741(0.560-0.980)$ & 0.036 \\
\hline SII(>489.52 VS. $\leq 489.52)$ & $3.919(3.087-4.977)$ & $<0.001$ & $2.707(2.074-3.533)$ & $<0.001$ \\
\hline Alb,g/L( $\leq 39.85$ VS. $>39.85)$ & $0.298(0.220-0.403)$ & $<0.001$ & $0.385(0.283-0.525)$ & $<0.001$ \\
\hline CEA,ug/ml(s2.17ug/ml VS. >2.17ug/ml) & $1.329(1.050-1.680)$ & 0.018 & $1.285(1.007-1.640)$ & 0.044 \\
\hline CA199,u/ml(s21u/mL VS. >21u/mL) & $1.560(1.208-2.013)$ & 0.001 & $1.184(0.905-1.550)$ & 0.218 \\
\hline $\mathrm{CA} 125, \mathrm{u} / \mathrm{ml}(\leq 14.6 \mathrm{u} / \mathrm{mll}$ VS. $>14.6 \mathrm{u} / \mathrm{ml})$ & $1.658(1.307-2.104)$ & $<0.001$ & $1.370(1.069-1.755)$ & 0.013 \\
\hline WBC $^{10} g^{\prime L}(\leq 6.525$ VS. $>6.525)$ & $2.158(1.698-2.742)$ & $<0.001$ & $1.361(1.047-1.770)$ & 0.021 \\
\hline Type of surgery & & $<0.001$ & & $<0.001$ \\
\hline DG & 1 (reference) & & 1 (reference) & \\
\hline PG & $1.781(1.325-2.395)$ & & $1.728(1.279-2.337)$ & \\
\hline TG & $2.331(1.736-3.129)$ & & $1.878(1.386-2.545)$ & \\
\hline TNM stage & & $<0.001$ & & 0.016 \\
\hline I & 1 (reference) & & 1 (reference) & \\
\hline$\|$ & $1.574(1.004-2.466)$ & & $1.248(0.788-1.978)$ & \\
\hline III & $2.385(1.665-3.416)$ & & $1.654(1.136-2.408)$ & \\
\hline
\end{tabular}


Table 3

Risk factors for the prognosis of GC patients after PSM

\begin{tabular}{|c|c|c|c|c|}
\hline \multirow[t]{2}{*}{ Parameters } & \multicolumn{2}{|l|}{ Univariate analysis } & \multicolumn{2}{|l|}{ Multutivariate analysis } \\
\hline & $\mathrm{HR}(95 \% \mathrm{Cl})$ & $\mathbf{P}$ & $\mathrm{HR}(95 \% \mathrm{Cl})$ & $\mathbf{P}$ \\
\hline $\operatorname{Age,y}(\leq 60$ VS. >60) & $1.544(1.121-2.126)$ & 0.008 & $1.356(0.973-1.889)$ & 0.072 \\
\hline Gender(male VS. female) & $0.958(0.649-1.415)$ & 0.83 & & \\
\hline Smoking(yes VS. no) & $1.023(0.744-1.407)$ & 0.887 & & \\
\hline Drinking(yes VS. no) & $1.098(0.790-1.527)$ & 0.577 & & \\
\hline Tumor size,mm( $\leq 5$ VS. >5) & $1.729(1.237-2.417)$ & 0.001 & $1.493(1.043-2.136)$ & 0.029 \\
\hline Differentiation(Poorly VS. Well and Moderately) & $0.733(0.514-1.046)$ & 0.087 & & \\
\hline SII(>489.52 VS. $\leq 489.52)$ & $2.472(1.757-3.480)$ & $<0.001$ & $2.669(1.881-3.788)$ & $<0.001$ \\
\hline Alb,g/L( $\leq 39.85$ VS. >39.85) & $0.306(0.198-0.474)$ & $<0.001$ & $0.286(0.183-0.447)$ & $<0.001$ \\
\hline CEA,ug/ml( $=2.17 \mathrm{ug} / \mathrm{ml} \mathrm{VS.}>2.17 \mathrm{ug} / \mathrm{ml})$ & $1.380(0.999-1.906)$ & 0.051 & & \\
\hline $\mathrm{CA} 199, \mathrm{u} / \mathrm{ml}(\leq 21 \mathrm{u} / \mathrm{mL}$ VS. $>21 \mathrm{u} / \mathrm{mL})$ & $1.415(0.996-2.011)$ & 0.053 & & \\
\hline $\mathrm{CA} 125, \mathrm{u} / \mathrm{ml}(\leq 14.6 \mathrm{u} / \mathrm{mll}$ VS. $>14.6 \mathrm{u} / \mathrm{ml})$ & $1.479(1.067-2.050)$ & 0.019 & $1.402(1.002-1.961)$ & 0.048 \\
\hline WBC $^{109 / L}(\leq 6.525$ VS. $>6.525)$ & $0.921(0.659-1.288)$ & 0.631 & & \\
\hline Type of surgery & & 0.001 & & 0.001 \\
\hline DG & 1 (reference) & & 1(reference) & \\
\hline PG & $1.803(1.159-2.805)$ & & $2.061(1.314-3.232)$ & \\
\hline TG & $2.230(1.451-3.428)$ & & $2.324(1.490-3.623)$ & \\
\hline TNM stage & & $<0.001$ & & 0.002 \\
\hline I & 1 (reference) & & 1 (reference) & \\
\hline ॥ & $1.803(1.159-2.805)$ & & $1.120(0.579-2.167)$ & \\
\hline III & $2.230(1.451-3.428)$ & & $2.123(1.257-3.584)$ & \\
\hline
\end{tabular}

\section{Clinicopathological Characteristics Of Age-stratified Patients After Psm}

After PSM, 199 non-elderly patients (56.21\%) and 155 elderly patients (43.79\%) were obtained for analysis. It was proved that SII was related to the TNM stage of non-elderly patients $(P<0.05)$ (Table 4). 
Age-stratified PSM

\begin{tabular}{|c|c|c|c|c|c|c|c|c|}
\hline \multirow[t]{2}{*}{ Parameters } & \multicolumn{4}{|c|}{ Non-elderly patients $(n=199)$} & \multicolumn{4}{|c|}{ Elderly patients( $n=155)$} \\
\hline & H-SII(n = 100) & L-SII(n = 99) & $x^{2}$ & $\mathbf{P}$ & H-SII(n = 77) & L-SII(n= 78) & $x^{2}$ & $\mathbf{P}$ \\
\hline Gender & & & 0.106 & 0.744 & & & 0.102 & 0.749 \\
\hline Male & $80(80.00 \%)$ & $81(81.82 \%)$ & & & $58(75.32 \%)$ & $57(73.08 \%)$ & & \\
\hline Female & $20(20.00 \%)$ & $18(18.18 \%)$ & & & $19(24.68 \%)$ & $21(26.92 \%)$ & & \\
\hline Smoking & & & 0.245 & 0.621 & & & 0.516 & 0.472 \\
\hline Yes & $47(47.00 \%)$ & $50(50.51 \%)$ & & & $37(48.05 \%)$ & $33(42.31 \%)$ & & \\
\hline No & $53(53.00 \%)$ & $49(49.49 \%)$ & & & $40(51.95 \%)$ & $45(57.69 \%)$ & & \\
\hline Drinking & & & 0.445 & 0.505 & & & 1.145 & 0.285 \\
\hline Yes & $42(42.00 \%)$ & $37(37.37 \%)$ & & & $30(38.96 \%)$ & $24(31.17 \%)$ & & \\
\hline No & $58(58.00 \%)$ & $62(62.63 \%)$ & & & $47(61.04 \%)$ & $54(70.13 \%)$ & & \\
\hline Tumor size (mm) & & & 0.834 & 0.361 & & & 0.102 & 0.749 \\
\hline$\leq 50$ & $70(70.00 \%)$ & $75(75.76 \%)$ & & & $58(75.32 \%)$ & $57(73.08 \%)$ & & \\
\hline$>50$ & $30(30.00 \%)$ & $24(24.24 \%)$ & & & $19(24.68 \%)$ & $21(26.92 \%)$ & & \\
\hline Differentiation & & & 0.065 & 0.799 & & & 0.073 & 0.787 \\
\hline Well and Moderately & $33(33.00 \%)$ & $68(68.69 \%)$ & & & $49(63.64 \%)$ & $48(61.54 \%)$ & & \\
\hline poorly & $67(67.00 \%)$ & $31(31.31 \%)$ & & & $28(36.36 \%)$ & $30(38.46 \%)$ & & \\
\hline Albumin (g/L) & & & 0.056 & 0.813 & & & 0.113 & 0.737 \\
\hline$\leq 39.85$ & $61(61.00 \%)$ & $62(62.63 \%)$ & & & $60(77.92 \%)$ & $59(75.64 \%)$ & & \\
\hline >39.85 & $39(39.00 \%)$ & $37(37.37 \%)$ & & & $17(22.08 \%)$ & $19(24.36 \%)$ & & \\
\hline $\mathrm{WBC}, 10^{9} / \mathrm{L}$ & & & 0.411 & 0.521 & & & 0.052 & 0.820 \\
\hline$\leq 6.525$ & $65(65.00 \%)$ & $60(60.61 \%)$ & & & $53(68.83 \%)$ & $55(70.51 \%)$ & & \\
\hline$>6.525$ & $35(35.00 \%)$ & $39(39.39 \%)$ & & & $24(31.17 \%)$ & $23(29.49 \%)$ & & \\
\hline CEA & & & 0.047 & 0.828 & & & 0.78 & 0.377 \\
\hline$\leq 7 \mathrm{ug} / \mathrm{ml}$ & $48(48.00 \%)$ & $46(46.46 \%)$ & & & $41(53.25 \%)$ & $36(46.15 \%)$ & & \\
\hline$>7 \mathrm{ug} / \mathrm{ml}$ & $52(52.00 \%)$ & $53(53.54 \%)$ & & & $36(46.75 \%)$ & $42(53.85 \%)$ & & \\
\hline CA199 & & & 0.085 & 0.771 & & & 0.773 & 0.379 \\
\hline$\leq 21 \mathrm{u} / \mathrm{mL}$ & $75(75.00 \%)$ & $76(76.77 \%)$ & & & $60(77.92 \%)$ & $56(71.79 \%)$ & & \\
\hline$>21 \mathrm{u} / \mathrm{mL}$ & $25(25.00 \%)$ & $23(23.23 \%)$ & & & $17(22.08 \%)$ & $22(28.21 \%)$ & & \\
\hline CA125 & & & 3.336 & 0.068 & & & 0.01 & 0.921 \\
\hline$\leq 14.6 \mathrm{u} / \mathrm{ml}$ & $65(65.00 \%)$ & 76(76.77\%) & & & $47(61.04 \%)$ & $47(60.26 \%)$ & & \\
\hline$>14.6 u / m l$ & $35(35.00 \%)$ & $23(23.23 \%)$ & & & $30(38.96 \%)$ & $31(39.74 \%)$ & & \\
\hline Type of surgery & & & 2.206 & 0.332 & & & 3.085 & 0.214 \\
\hline DG & $36(36.00 \%)$ & $26(26.26 \%)$ & & & $21(27.27 \%)$ & $13(16.67 \%)$ & & \\
\hline
\end{tabular}




\begin{tabular}{|c|c|c|c|c|c|c|c|c|}
\hline \multirow[t]{2}{*}{ Parameters } & \multicolumn{4}{|c|}{ Non-elderly patients $(n=199)$} & \multicolumn{4}{|c|}{ Elderly patients $(n=155)$} \\
\hline & H-SII $(n=100)$ & L-S॥( $(n=99)$ & $x^{2}$ & $\mathbf{P}$ & $\mathrm{H}-\mathrm{SII}(\mathrm{n}=77)$ & L-SII $(n=78)$ & $x^{2}$ & $\mathbf{P}$ \\
\hline PG & $32(32.00 \%)$ & $36(36.37 \%)$ & & & $27(35.07 \%)$ & $27(34.62 \%)$ & & \\
\hline TG & $32(32.00 \%)$ & $37(37.37 \%)$ & & & $29(37.66 \%)$ & $38(48.71 \%)$ & & \\
\hline TNM stage & & & 14.683 & 0.001 & & & 4.102 & 0.129 \\
\hline I & $14(14.00 \%)$ & $18(18.18 \%)$ & & & $16(20.78 \%)$ & $17(21.79 \%)$ & & \\
\hline II & $9(9.00 \%)$ & $28(28.28 \%)$ & & & $22(28.57 \%)$ & $12(15.38 \%)$ & & \\
\hline III & $77(77.00 \%)$ & $53(53.54 \%)$ & & & $39(50.65 \%)$ & $49(62.83 \%)$ & & \\
\hline
\end{tabular}

Cox regression analysis on influencing factors for the prognosis of non-elderly patients

The results of univariate analysis showed that the maximum diameter of tumor, SII, ALb, CA125 and TNM stage were risk factors for the prognosis of the 199 non-elderly patients with GC. The results of multivariate analysis showed that SII $(\mathrm{HR}=3.383,95 \% \mathrm{Cl}$ : $1.961-5.838, \mathrm{P}<0.001)$ and $\mathrm{ALb}(\mathrm{HR}=0.400,95 \% \mathrm{Cl}: 0.238-0.674, \mathrm{P}=0.001)$ were independent risk factors for the prognosis of non-elderly patients (Table 5). 
Table 5

Univariate and multivariate analyses on OS of non-elderly patients

\begin{tabular}{|c|c|c|c|c|}
\hline \multirow[t]{2}{*}{ Parameters } & \multicolumn{2}{|l|}{ Univariate analysis } & \multicolumn{2}{|c|}{ Multutivariate analysis } \\
\hline & $\mathrm{HR}(95 \% \mathrm{Cl})$ & $P$ & $\mathrm{HR}(95 \% \mathrm{Cl})$ & $P$ \\
\hline Gender $\gtrsim m a l e ~ V S$. female囚 & $0.834 \rrbracket 0.459-1.516 \rrbracket$ & 0.552 & & \\
\hline Smoking $\varangle$ yes VS. no $\rrbracket$ & $1.042 \varangle 0.663-1.639 \rrbracket$ & 0.858 & & \\
\hline Drinking $\rrbracket y e s ~ V S$. no® & $1.090 \rrbracket 0.688-1.726 \rrbracket$ & 0.714 & & \\
\hline Tumor size,mm $\rrbracket \leq 5$ VS. $>5 \rrbracket$ & $1.878 \rrbracket 1.177-2.995 \rrbracket$ & 0.008 & $1.452 ه 0.893-2.359 \rrbracket$ & 0.133 \\
\hline 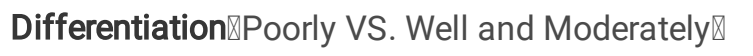 & $0.926 \rrbracket 0.561-1.528 \rrbracket$ & 0.764 & & \\
\hline SII $\backslash>489.52$ VS. $\leq 489.52 \rrbracket$ & $3.970 \rrbracket 2.334-6.751 \rrbracket$ & $<0.001$ & $3.383 \rrbracket 1.961-5.838 \rrbracket$ & $<0.001$ \\
\hline Albumin,g/L $₫ \leq 39.85$ VS. $>39.85 \rrbracket$ & $0.462 \varangle 0.277-0.769 \rrbracket$ & 0.003 & $0.400 \rrbracket 0.238-0.674 \rrbracket$ & 0.001 \\
\hline 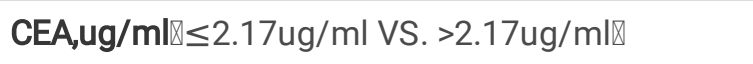 & $1.208 \bowtie 0.768-1.901 \rrbracket$ & 0.413 & & \\
\hline $\mathrm{CA} 199, \mathrm{u} / \mathrm{ml} \| \leq 21 \mathrm{u} / \mathrm{mL}$ VS. $>21 \mathrm{u} / \mathrm{mL} \rrbracket$ & $1.110 \rrbracket 0.660-1.867 \rrbracket$ & 0.693 & & \\
\hline $\mathrm{CA} 125, \mathrm{u} / \mathrm{ml} \| \leq 14.6 \mathrm{u} / \mathrm{mll} \mathrm{VS} .>14.6 \mathrm{u} / \mathrm{ml} \rrbracket$ & $1.783 \rrbracket 1.124-2.830 \rrbracket$ & 0.014 & $1.592 \varangle 0.992-2.554 \rrbracket$ & 0.054 \\
\hline WBC,109/L $₫ \leq 6.525$ VS. $>6.525 \rrbracket$ & $0.981 \otimes 0.616-1.561 \rrbracket$ & 0.934 & & \\
\hline Type of surgery & & 0.104 & & \\
\hline DG & 1هreference $\rrbracket$ & & & \\
\hline PG & $1.801 \rrbracket 1.039-3.123 \rrbracket$ & & & \\
\hline TG & $1.296 \rrbracket 0.723-2.324 \rrbracket$ & & & \\
\hline TNM stage & & 0.004 & & 0.090 \\
\hline I & 1邓reference》 & & 1邓reference $\rrbracket$ & \\
\hline ॥ & $1.644 \rrbracket 0.549-4.920 \rrbracket$ & & $2.111 \otimes 0.686-6.493 \rrbracket$ & \\
\hline III & $3.539 \otimes 1.423-8.799 \rrbracket$ & & $2.776 \rrbracket 1.091-7.063 \rrbracket$ & \\
\hline
\end{tabular}

Cox regression analysis on influencing factors for the prognosis of elderly patients

The results of univariate analysis showed that the histological type, SII, ALb, CEA, CA199, operation method and TNM stage were risk factors for the prognosis of the 155 elderly patients with $\mathrm{GC}$. It was confirmed by multivariate analysis that $\mathrm{SII}(\mathrm{HR}=2.372$, $95 \% \mathrm{Cl}: 1.444-3.896, \mathrm{P}=0.001), \mathrm{ALb}(\mathrm{HR}=0.164,95 \% \mathrm{Cl}: 0.059-0.457, \mathrm{P}=0.001)$ and operation method were independent risk factors for the prognosis of elderly patients (Table 6). 
Table 6

Univariate and multivariate analyses on OS of elderly patients

\begin{tabular}{|c|c|c|c|c|}
\hline \multirow[t]{2}{*}{ Parameters } & \multicolumn{2}{|l|}{ Univariate analysis } & \multicolumn{2}{|l|}{ Multutivariate analysis } \\
\hline & $\mathrm{HR}(95 \% \mathrm{Cl})$ & $P$ & $\mathrm{HR}(95 \% \mathrm{Cl})$ & $P$ \\
\hline Gender $\Downarrow$ male VS. female》 & $0.976 \varangle 0.574-1.661 \rrbracket$ & 0.928 & & \\
\hline 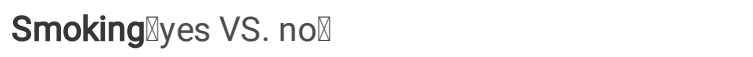 & $0.960 \otimes 0.610-1.513 \rrbracket$ & 0.861 & & \\
\hline Drinking $\rrbracket y e s ~ V S$. no $\rrbracket$ & $1.024 \rrbracket 0.635-1.652 \rrbracket$ & 0.922 & & \\
\hline Tumor size,mm $₫ \leq 5$ VS. $>5 \rrbracket$ & $1.610 \otimes 0.993-2.611 \rrbracket$ & 0.053 & & \\
\hline 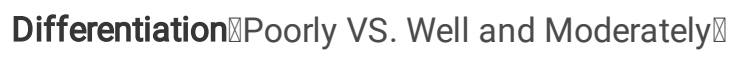 & $0.532 \varangle 0.321-0.881 \rrbracket$ & 0.014 & $0.689 \rrbracket 0.410-1.158 \rrbracket$ & 0.160 \\
\hline SII $\backslash>489.52$ VS. $\leq 489.52 \rrbracket$ & $1.612 \otimes 1.012-2.567 \rrbracket$ & 0.044 & $2.372 \otimes 1.444-3.896 \rrbracket$ & 0.001 \\
\hline Alb,g/L $₫ \leq 39.85$ VS. $>39.85 \rrbracket$ & $0.132 \varangle 0.048-0.361 \rrbracket$ & $<0.001$ & $0.164 \rrbracket 0.059-0.457 \rrbracket$ & 0.001 \\
\hline 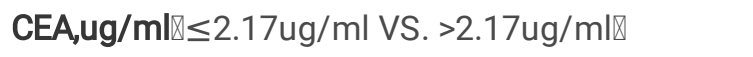 & $1.704 \rrbracket 1.069-2.715 \rrbracket$ & 0.025 & $1.530 \rrbracket 0.930-2.517 \rrbracket$ & 0.094 \\
\hline $\mathrm{CA} 199, \mathrm{u} / \mathrm{ml} \otimes \leq 21 \mathrm{u} / \mathrm{mL}$ VS. $>21 \mathrm{u} / \mathrm{mL} \mathbb{}$ & $1.848 \rrbracket 1.138-3.002 \rrbracket$ & 0.013 & $1.457 \rrbracket 0.877-2.421 \rrbracket$ & 1.146 \\
\hline $\mathrm{CA} 125, \mathrm{u} / \mathrm{ml} \| \leq 14.6 \mathrm{u} / \mathrm{mll} \mathrm{VS} .>14.6 \mathrm{u} / \mathrm{ml} \rrbracket$ & $1.142 \varangle 0.718-1.818 \rrbracket$ & 0.574 & & \\
\hline WBC,109/L $₫ \leq 6.525$ VS. $>6.525 \rrbracket$ & $0.940 \otimes 0.574-1.539 \rrbracket$ & 0.806 & & \\
\hline Type of surgery & & $<0.001$ & & 0.002 \\
\hline DG & 1『reference $\rrbracket$ & & 1هreference区 & \\
\hline PG & $1.893 \rrbracket 0.875-4.098 \rrbracket$ & & $1.548 \rrbracket 0.709-3.381 \rrbracket$ & \\
\hline TG & $4.004 \rrbracket 1.937-8.279 \rrbracket$ & & $3.174 \rrbracket 1.490-6.760 \rrbracket$ & \\
\hline TNM stage & & 0.020 & & 0.090 \\
\hline I & 1囚reference》 & & 1》reference》 & \\
\hline ॥ & $0.902 \varangle 0.397-2.049 \rrbracket$ & & $0.766 \rrbracket 0.328-1.786 \rrbracket$ & \\
\hline III & $1.914 \rrbracket 1.020-3.590 \rrbracket$ & & $1.539 \llbracket 0.798-2.967 \rrbracket$ & \\
\hline
\end{tabular}

\section{Postoperative Survival Rate Of Non-elderly And Elderly Patients}

Among the non-elderly patients, the 1-, 3- and 5-year survival rates were $92.9 \%, 82.8 \%$ and $62.1 \%$, respectively, in L-SII group, and $85.0 \%, 47.6 \%$ and $31.9 \%$, respectively, in H-SII group. The survival rate of GC patients in L-SII group was better than that in $\mathrm{H}-\mathrm{SII}$ group, and the OS rate had a statistically significant difference between the two groups $(P<0.001)$. Among the elderly patients, the 1-, 3- and 5-year survival rates were $83.3 \%, 63.2 \%$ and $43.8 \%$, respectively, in L-SIl group, and $75.3 \%, 42.2 \%$ and $19.4 \%$, respectively, in H-SII group. The survival rate of GC patients in L-SIl group was better than that in H-SIl group, and the OS rate had a statistically significant difference between the two groups $(P=0.041)$ (Fig. 5).

\section{Discussion}

In recent years, related studies have demonstrated that the inflammatory response (tissue necrosis, activation of immune response, secretion of cytokines) plays an important role in the occurrence, development and metastasis of tumors ${ }^{[15,16]}$. In the early stage, the inflammatory response is induced and the anti-tumor immune response is inhibited, thereby promoting growth of 
tumor cells. With the growth of tumor, the inflammatory response is further enhanced. To eliminate inflammation, the recruitment of immune cells to tumor tissues is reduced, resulting in immune escape of tumor cells. In the late stage, the body's immunity is almost lost, and the inflammatory response is further worsened, thus facilitating the tumor progression [17]. SII, a new inflammatory index integrating the NE, LY and PLT counts ${ }^{[18]}$, can reflect the balance status between tumor immunity and inflammatory response more comprehensively than platelet-to-lymphocyte ratio (PLR), neutrophil-to-lymphocyte ratio (NLR) and monocyte-to-lymphocyte ratio (MLR), and better predict the prognosis of cancer patients $[18,19,20]$.

In the present study, the optimal cut-off value of SII was determined using the ROC curve, based on which the patients were divided into H-SII group and low L-SII group. In terms of the correlation between SII and the clinicopathological characteristics of patients, SIl was related to the maximum diameter of tumor, histological type, ALb, WBC, CA125 and TNM stage $(P<0.05)$. In addition, the survival time of GC patients in H-SII group was significantly shortened, and the 5-year survival rate was significantly lower than that in L-SII group. Wang ${ }^{[21]}$ et al. retrospectively analyzed the clinical data of $444 \mathrm{GC}$ patients treated with gastrectomy and found that the 5 -year survival rate is $29.4 \%$ and $46.1 \%$, respectively, in H-SIl group and L-SII group, suggesting that the SII level is related to the prognosis of GC patients, consistent with the results of this study. Han BL ${ }^{[22]}$ also showed that SII is closely related to the prognosis of GC patients, and high SIl indicates a poor prognosis of GC patients. Its mechanism can be explained by the functions of NE, PLT and LY. In the case of tumor invasion, the inflammatory response can promote the increase in NE and the secretion of NO, reactive oxygen species, arginase and other active substances, which can regulate the activity of NK cells and LY, thereby facilitating tumor growth and metastasis ${ }^{[23,24]}$. In addition, the PLT count rises in inflammation, and PLT promotes tumor angiogenesis via releasing vascular endothelial growth factor and platelet-derived growth factor ${ }^{[25]}$, creating favorable conditions for tumor growth. Moreover, PLT can form colonizing tumor thrombi with tumor cells, thus promoting the further invasion of tumor cells into the body. On the contrary, LY can effectively suppress the occurrence and development of tumors, and induce the death of tumor cells. The long-term inflammatory stimulus will lead to decline in LY subsets, thereby causing immune dysfunction, and the decrease in LY count can raise the risk of immune escape of tumor cells $[26,27]$. Therefore, SII can reflect the relation between systemic inflammatory response and body's immunity during tumor progression. The higher the SII is, the severer the inflammatory response and immunosuppression in GC patients will be.

In this study, it was confirmed by univariate analysis before and after PSM that age, maximum diameter of tumor, SII, ALb, CA125, operation method and TNM stage were risk factors for the prognosis of GC patients. Before PSM, the results of multivariate analysis showed that age, histological type, SII, ALb, CEA, CA125, WBC, operation method and TNM stage were independent risk factors for the prognosis of GC patients. After PSM, the results of multivariate analysis revealed that the maximum diameter of tumor, SII, ALb, CA125, operation method and TNM stage were independent risk factors for the prognosis of GC patients. Then the statistically significant factors after PSM (except SII) were analyzed. It was found that the larger maximum diameter of tumor, lower level of ALb, higher level of CA125 and higher TNM stage indicated a worse prognosis. The prognosis was better among patients undergoing DG than that among patients treated with PG and TG. Wang et al. ${ }^{[28]}$ found in the study on the correlation between SII and the prognosis of GC patients that SII and TNM stage are independent risk factors for the prognosis of GC patients, consistent with the results of this paper. As the main component of plasma protein, ALb can reflect the nutritional status of patients, and it has also been widely used as an antidote and transporter in predicting the survival status and disease progression of cancer patients ${ }^{[29,30]}$. CA125 is mainly present in epithelial tissues and serum of patients, and its expression level in the serum of GC patients is higher than that in normal people, which is positively correlated with the TNM stage and negatively correlated with the prognosis of GC patients, consistent with the results of this study. This indicates that CA125 can be used to assess the severity of GC ${ }^{[31]}$. In addition, the multivariate analysis before and after PSM demonstrated that no positive result was obtained in age after PSM, but other scholars argued that there is a correlation between age and the prognosis of GC patients ${ }^{[32,}$ 33]. The reasons why age was not an independent risk factor for the prognosis of GC patients in this study are as follows: PSM was used in this study to validate the impact of SII on the prognosis on the basis of excluding other confounding factors, which stressed the effect of SII on the prognosis, thus affecting the prognostic assessment by other indexes to a certain extent. Besides, it is possibly because the sample size was small after PSM and this study itself had limitations, rather than simply considering age unrelated to the prognosis of patients. Therefore, the sample size remains to be further expanded in the future. To further explore the correlation between age and the prognosis of GC patients, age was further subjected to stratification analysis to exclude the value deviation in a certain stage caused by the small sample size or the bias of age stratification in this study. It is

Page $13 / 20$ 
reported in the literature that elderly patients are aged $\geq 60$ years old ${ }^{[34,35]}$. In this study, the median age of 354 patients was 59.0 years old after PSM. Therefore, patients aged $\geq 60$ years old were defined as elderly patients in this study. The results revealed that the mean SII of elderly patients (495.73) was significantly higher than that of non-elderly patients (443.21), suggesting the correlation between SII and patient's age. In addition, the risk factors for the prognosis of GC patients were analyzed under age stratification. It was found that the high SIl was correlated with the poor prognosis of elderly patients with GC, and the 5-year OS rate of elderly patients was lower than that of non-elderly patients in H-SIl group (31.9\% vs. $62.1 \%)$. The specific mechanism remains to be further confirmed by a large number of prospective studies.

There were certain limitations in this study. First, this was a small-sample retrospective study on GC patients in high-altitude areas. To better control the bias, PSM was used for data analysis, but all the impact of covariates on the outcome failed to be fully eliminated. Second, this was a single-center study, so the conclusion cannot fully represent the characteristics of other study centers and populations, limiting its popularization. Therefore, the preliminary results need to be validated by larger-sample randomized controlled studies in the future, so as to offer more convincing theoretical support to the existing conclusion.

In conclusion, SII, that remains simple, universal, non-invasive, cheap and reproducible, is expected to be an index for assessing the prognosis of GC patients.

\section{List Of Abbreviations}

\begin{tabular}{|ll|}
\hline systemic immune inflammation index & SII \\
\hline gastric carcinoma & GC \\
\hline receiver operating characteristic & ROC \\
\hline propensity score matching & PSM \\
\hline high SII & H-SII \\
\hline low SII & L-SII \\
\hline hazard ratio & HR \\
\hline confidence interval & Cl \\
\hline neutrophil & NE \\
\hline lymphocyte & LY \\
\hline platelet & PLT \\
\hline distal gastrectomy & DG \\
\hline proximal gastrectomy & PG \\
\hline total gastrectomy & TG \\
\hline Overall survival & OS \\
\hline albumin & ALb \\
\hline white blood cell & WBC \\
\hline carbohydrate antigen 125 & CA125 \\
\hline carcinoembryonic antigen & CEA \\
\hline platelet-to-lymphocyte ratio & PLR \\
\hline neutrophil-to-lymphocyte ratio & NLR \\
\hline monocyte-to-lymphocyte ratio & MLR \\
\hline
\end{tabular}

Page $14 / 20$ 


\section{Declarations}

\section{Author Contributions $\square$}

Xu Zhaojun and Chen Xiaobin: Designed the research and wrote the paper; Chen Xiaobin, Jiang Shuyun, Liu Tao and Wang Cheng: Revised the paper; Xu Zhaojun, Cai Baojia and Chen Xiaobin: Participated in research work; Xu Zhaojun, Chen Xiaobin: Collected samples; Xu Zhaojun, Chen Xiaobin and Yuan Jiaqi : Analyzed data and constructed figures; Ma Xiaoming and An Juan : Responsible for project guidance.

\section{Conflicts of Interest and Source of Funding:}

Drs. Xu Zhaojun, Chen Xiaobin, An Juan, Yuan Jiaqi, Jiang Shuyun, Liu Tao, Cai Baojia, Wang Chengand Ma Xiaoming have no conflicts of interest or financial ties to disclose.

This work was supported by the Key R \& D and transformation program of Qinghai Province-Special project of science and technology assistance (No.2021-QY-213); the CAS (Light of the West China) Program (No.2019-33), the National Natural Science Foundation of China (No.81460429), the Open Project of State Key Laboratory of Plateau Ecology and Agriculture, Qinghai University (No.2019-ZZ-07), the Scientific Research Project of Research Team of Medical College of Qinghai University (No. 2020KYT-2)

\section{Data availability}

The datasets generated during and/or analysed during the current study are available from the corresponding author on reasonable request.

\section{Acknowledgements}

Research reported in this publication was supported by grant number 81460429 from the National Natural Science Foundation of China.

\section{Additional Information}

\section{Ethical approval}

Ethical approval was not required in the treatment of the patient in this report.

\section{Consent}

Written consent has been received from the subject.

\section{References}

1 Freddie Bray BSc, MSc, PhD et al. Global cancer statistics 2018: GLOBOCAN estimates of incidence and mortality worldwide for 36 cancers in 185 countries[J]. CA: A Cancer Journal for Clinicians, 2018, 68(6) : 394-424.

2 Kun Gao and Jun Wu. National trend of gastric cancer mortality in China (2003-2015): a population-based study[J]. $2019,39(1): 24$.

Park, Lee, HoJae, et al. Overview of gastrointestinal cancer prevention in Asia[C]// Asia-pacific Microwave Conference. 2015.

4 Prete A D , Allavena P, Santoro G , et al. Molecular pathways in cancer-related inflammation[J]. Biochemia Medica, 2011, 21(3):264-275.

5 Cancer-Related Inflammation[J]. Journal of Clinical Immunology, 2013, 33(1):79-84 
6 The pretreatment neutrophil-to-lymphocyte ratio predicts therapeutic response to radiation therapy and concurrent chemoradiation therapy in uterine cervical cancer.[J]. International Journal of Clinical Oncology, 2015, 20(5):989-996.

7 LABELLE M, BEGUM S, HYNES RO. Direct signaling between platelets and carcinoma cells induces an epithelialmesenchymal-like transition and promotes metastasis. 2011, 20®5®:576-90.

$8 \quad$ Zhu Lizhen et al. A new prognostic score based on the systemic inflammatory response in patients with inoperable nonsmall-cell lung cancer.[J]. OncoTargets and therapy, 2016, $9: 4879-86$.

9 GAO YB, GUO W, CAI SH, et al. Systemic immune-inflammation index $₫$ SII区 is useful to predict survival outcomes in patients with surgically resected esophageal squamous cell carcinoma. 2019, 10囚14囚:3188-3196.

10 WANG P, YUE WS, LI WY, et al. Systemic immune-inflammation index and ultrasonographic classification of breast imaging-reporting and data system predict outcomes of triple-negative breast carcinoma. 2019, 11:813-819.

11 HU B, YANG XR, XU Y, et al. Systemic immune-inflammation index predicts prognosis of patients after curative resection for hepatocellular carcinoma. 2014, 20冈23囚:6212-22.

12 AZIZ MH, SIDERAS K, AZIZ NA, et al. The Systemic-immune-inflammation Index Independently Predicts Survival and Recurrence in Resectable Pancreatic carcinoma and its Prognostic Value Depends on Bilirubin Levels: A Retrospective Multicenter Cohort Study. 2019, 270冈1邓:139-146.

13 Shan Fei, Li Ziyu, Zhang Lianhai, Li Shuangxi, Jia Yongning, Miao Rulin, Xue Kan, Li Zhemin, Gao Xiangyu, Wang Yinkui, Yan Chao, Li Shen, Ji Jiafu. The Union for International Cancer Control (UICC) and the American Joint Committee on Cancer (AJCC) gastric cancer TNM staging system ( $8^{\text {th }}$ edition) explanation and elaboration [J]. Chinese Journal of Practical Surgery, 2017,37(01): 15-17

14 Hu Xiang. Changes of Japanese "Guidelines" and New Trends of Gastric Cancer Treatment [J]. Chinese Journal of Practical Surgery, 2019,39(03): 231-236

15 Sergei I. Grivennikov, Florian R. Greten, Michael Karin. Immunity, Inflammation, and carcinoma. 2010, 140区6®:883-899.

16 Singh R, Mishra MK, Aggarwal H. Inflammation, Immunity, and Cancer. Mediators Inflamm. 2017;2017:6027305.

17 Huang B. Regulation of immune response and inflammation in tumor microenvironment[J]. Chinese Journal of Cancer Biotherapy, 2012,19(02):111-115.

18 Fu Hongyuan et al. Systemic Immune-Inflammation Index (SII) is Useful to Predict Survival Outcomes in Patients After Liver Transplantation for Hepatocellular Carcinoma within Hangzhou Criteria.[J]. Cellular physiology and biochemistry : international journal of experimental cellular physiology, biochemistry, and pharmacology, 2018, 47(1) : 293-301.

19 Diem S, Schmid S, Krapf M, Flatz L, Born D, Jochum W, Templeton AJ, Früh M. Neutrophil-to-Lymphocyte ratio (NLR) and Platelet-to-Lymphocyte ratio (PLR) as prognostic markers in patients with non-small cell lung cancer (NSCLC) treated with nivolumab. Lung Cancer. 2017 Sep;111:176-181.

20 Trifan G, Testai FD. Systemic Immune-Inflammation (SII) index predicts poor outcome after spontaneous supratentorial intracerebral hemorrhage. J Stroke Cerebrovasc Dis. 2020 Sep;29(9):105057.

21 Kang Wang,Feiyu Diao,Zhijun Ye,Xinhua Zhang,Ertao Zhai,Hui Ren,Tong Li,Hui Wu,Yulong He,Shirong Cai,Jianhui Chen.Prognostic value of systemic immune-inflammation index in patients with gastric carcinoma[J].Chinese Journal of carcinoma,2017,36ख09®:420-426.

22 Han BL, Wang YM, Xue YW. Effect of preoperative systemic immune-inflammation index on the prognosis of patients with gastric cancer[J]. Chinese Journal of General Surgery, 2019(04):306-309.

Page $16 / 20$ 
23 Hu XX, He YF, Luo HQ, Chen WJ, Ke LH, Yan Y, Wu SS, Niu JY, Li HM, Xu HJ, Hu B. The relationship between peripheral blood NLR, PLR and clinical prognosis of small cell esophageal cancer[J]. Chinese Clinical Oncology, 2019,24(06):543-547.

24 Teramukai S , Kitano T, Kishida Y , et al. Pretreatment neutrophil count as an independent prognostic factor in advanced non-small-cell lung cancer: An analysis of Japan Multinational Trial Organisation LC00-03[J]. European Journal of Cancer, 2009, 45(11):1950-1958.

25 Brill A, Dashevsky O, Rivo J, Gozal Y, Varon D. Platelet-derived microparticles induce angiogenesis and stimulate postischemic revascularization. Cardiovasc Res. 2005 Jul 1;67(1):30-8.

26 Hu Hong et al. Prognostic value of preoperative NLR, dNLR, PLR and CRP in surgical renal cell carcinoma patients.[J]. World journal of urology, 2017, 35(2) : 261-270.

27 Ozmen S et al. Neutrophil-lymphocyte ratio (NLR) and platelet-lymphocyte ratio (PLR) may be superior to C-reactive protein (CRP) for predicting the occurrence of differentiated thyroid cancer.[J]. Endocrine regulations, 2017, 51(3) : 131-136.

28 Wang Qingshan and Zhu Dayong. The prognostic value of systemic immune-inflammation index (SII) in patients after radical operation for carcinoma of stomach in gastric cancer.[J]. Journal of gastrointestinal oncology, 2019, 10(5) : 965-978.

29 Atsushi Abe et al. Correlation between prognostic nutritional index and occlusal status in gastric cancer[J]. Oral Diseases, 2020, 26(2) : 465-472.

30 Maria Mantzorou, Antonios Koutelidakis, Stamatios Theocharis, et al. Clinical Value of Nutritional Status in Cancer: What is its Impact and how it Affects Disease Progression and Prognosis? 2017, 69(8):1151-1176.

31 Huang Chao et al. Clinical Significance of Serum CA125, CA19-9, CA72-4, and Fibrinogen-to-Lymphocyte Ratio in Gastric Cancer With Peritoneal Dissemination.[J]. Frontiers in oncology, 2019, 9 : 1159.

32 Zou XN, Wan X, Dai Z, Yang GH. Epidemiological characteristics of cancer in elderly chinese. ISRN Oncol. 2012;2012:381849.

33 Su Yan,Bin Li,Zhen-Zhong Bai,Jun-Qi Wu,Da-Wei Xie,Ying-Cai Ma,Xu-Xiang Ma,Jun-Hui Zhao,Xin-Jian Guo.Clinical epidemiology of gastric cancer in Hehuang valley of China:A 10-year epidemiological study of gastric cancer[J]. World Journal of Gastroenterology,2014,20(30):10486-10494.

34 Yang LD, Wang XD, Li Q, Liang YH, Chen Y, Wu MJ, Xu F. The safety in more than 60-year-old gastric cancer patients treated by fast-track surgery[J]. Journal of Modern Oncology, 2015,23(19):2814-2817.

$35 \mathrm{Xu} \mathrm{PW}$, Zhang H. Clinical manifestations, pathological features and prognosis of elderly patients with gastric cancer[J]. Journal of Nanjing Medical University (Natural Science), 2015,35(03):398-400.

\section{Figures}




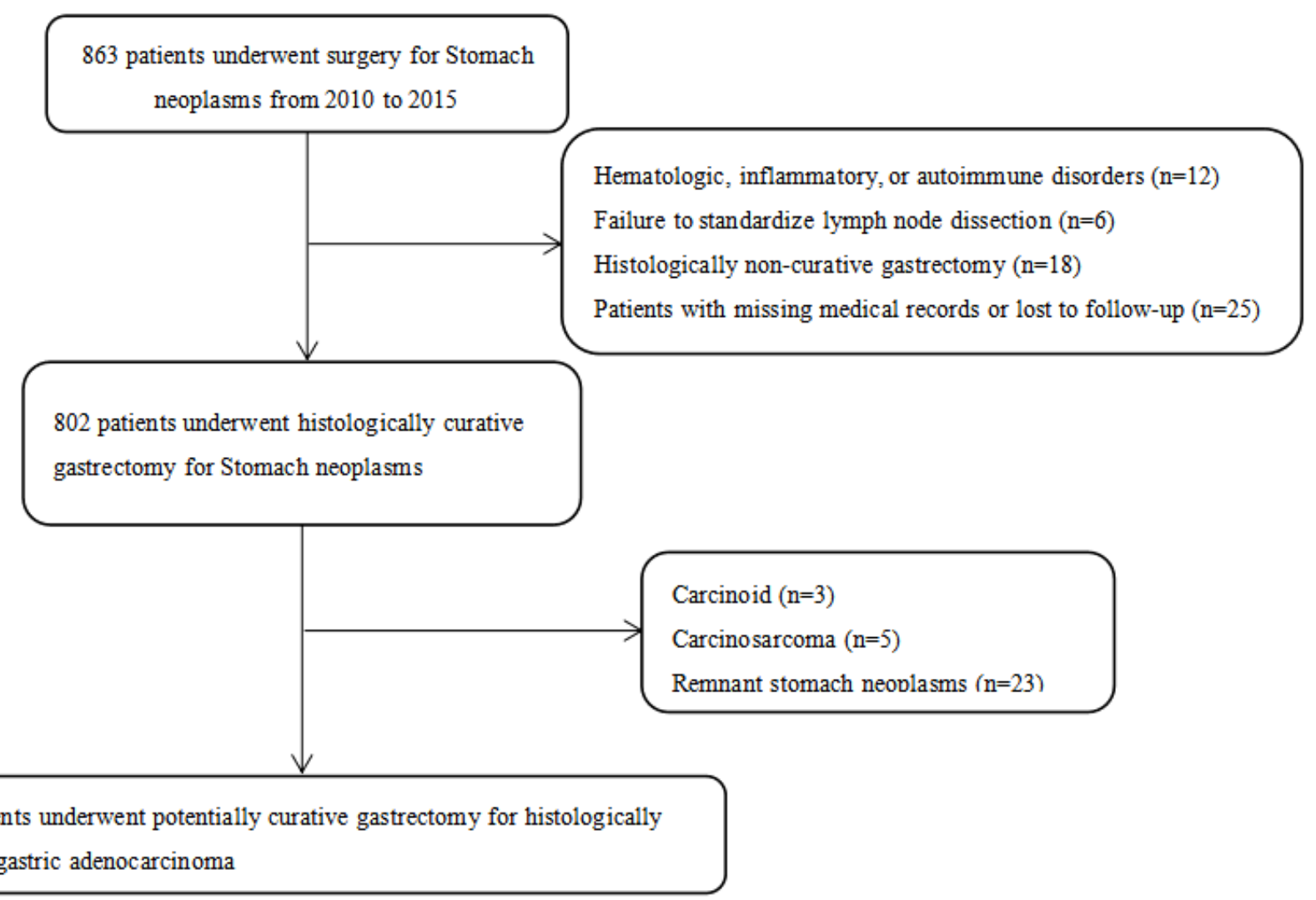

\section{Figure 1}

Study flow diagram.

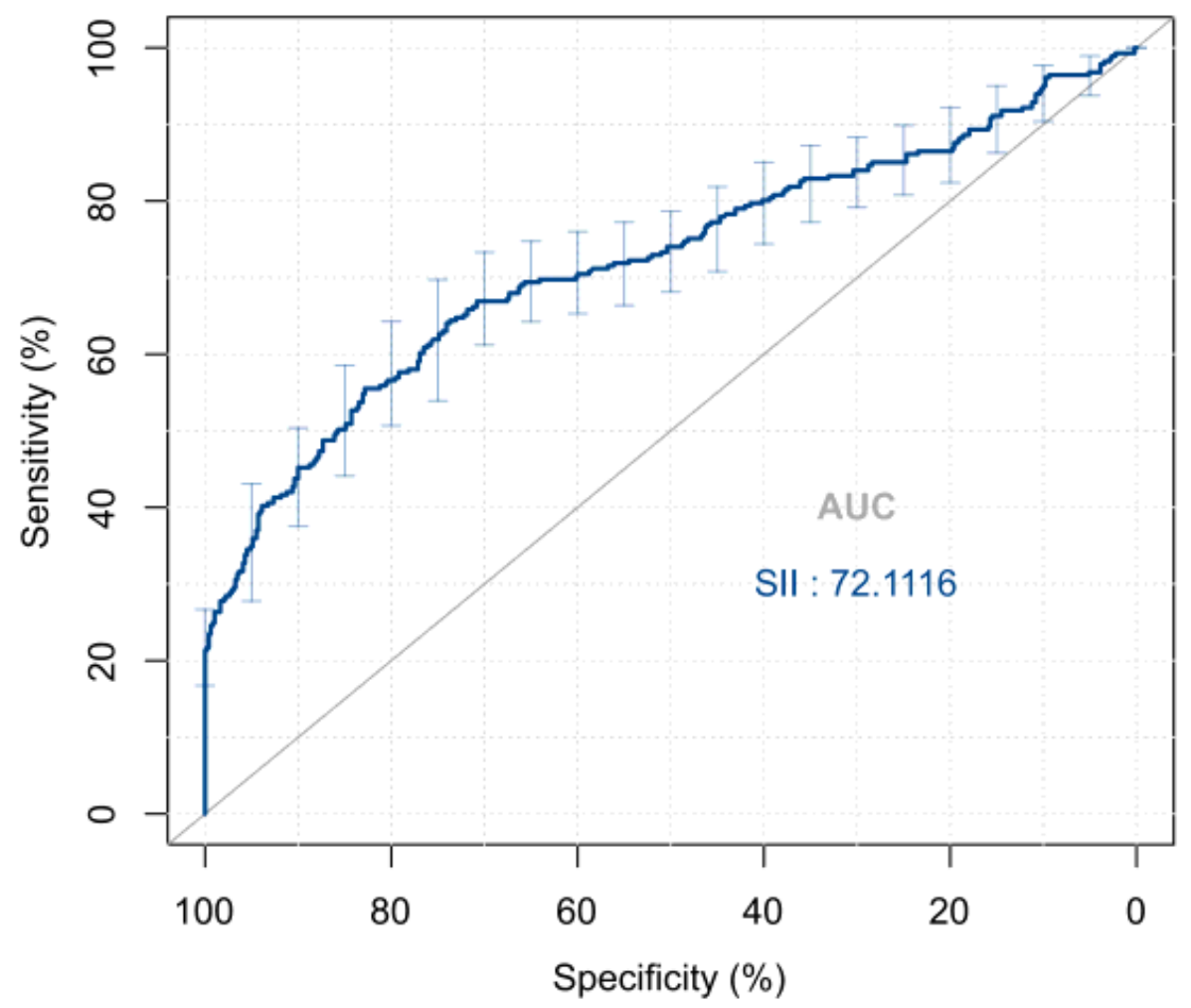


Figure 2

ROC curves were plotted to determine the optimal cut-off value of SII.

(a)

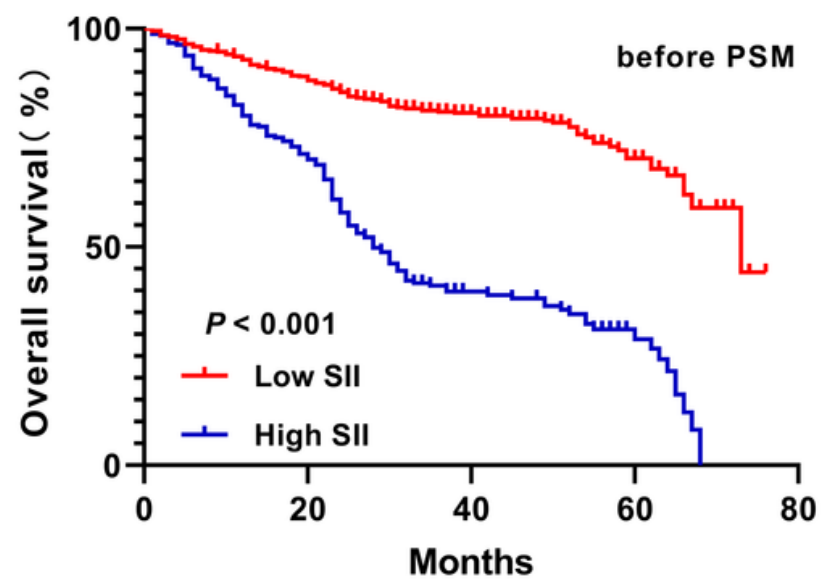

(b)

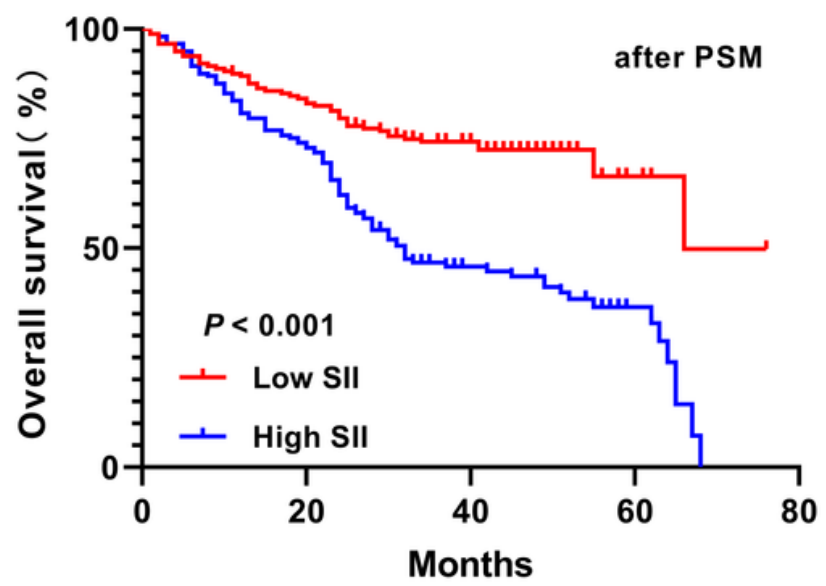

Figure 3

Postoperative OS curve in the two groups before and after PSM. a: Before PSM, the 5-year OS rate in L-SIl group was significantly higher than that in H-SIl group (67.9\% vs. 28.9\%) (P<0.001). b: After PSM, the 5-year OS rate in L-SIl group was significantly higher than that in $\mathrm{H}-\mathrm{SIl}$ group $(60.4 \%$ vs. $32.9 \%)(\mathrm{P}<0.001)$.

(a)

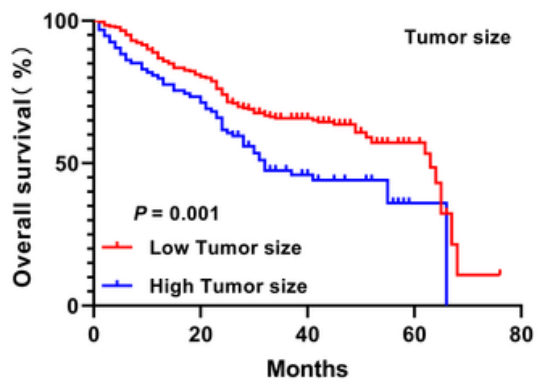

(b)

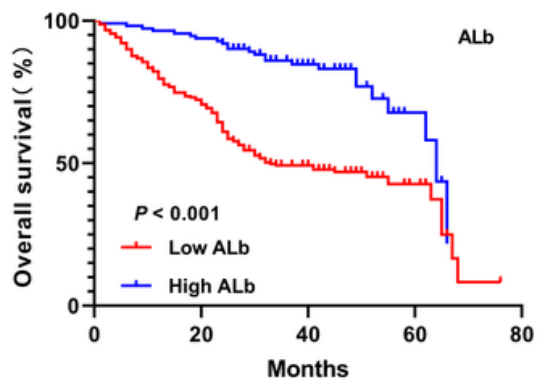

(c)

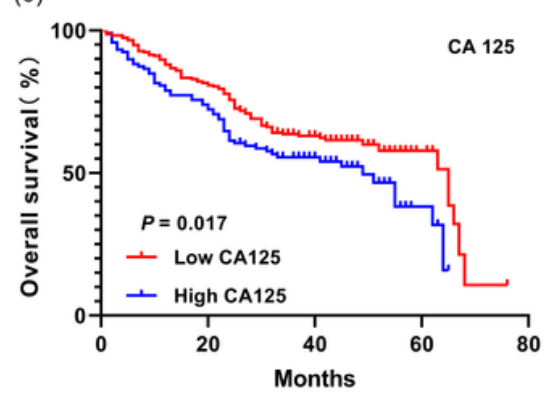

(d)

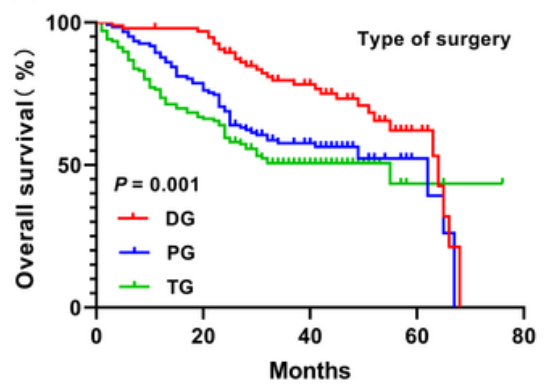

(e)

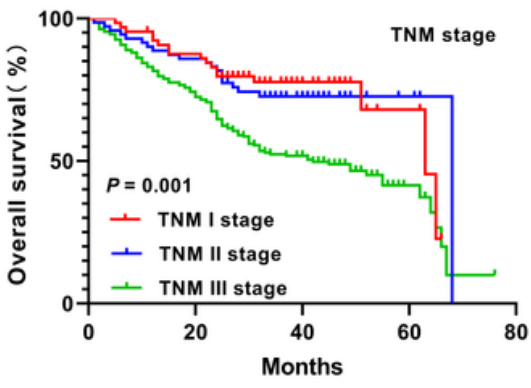

Figure 4

Survival curves under different risk factors for the prognosis after PSM. a: different tumor sizes (H-SIl group vs. L-SIl group). b: Different ALb levels (H-SIl group vs. L-SII group). c: Different CA125 levels (H-SIl group vs. L-SII group). d: Different operation methods (DG vs. PG vs. TG). e: Different TNM stages (stage I vs. stage II vs. stage III). 
(a)

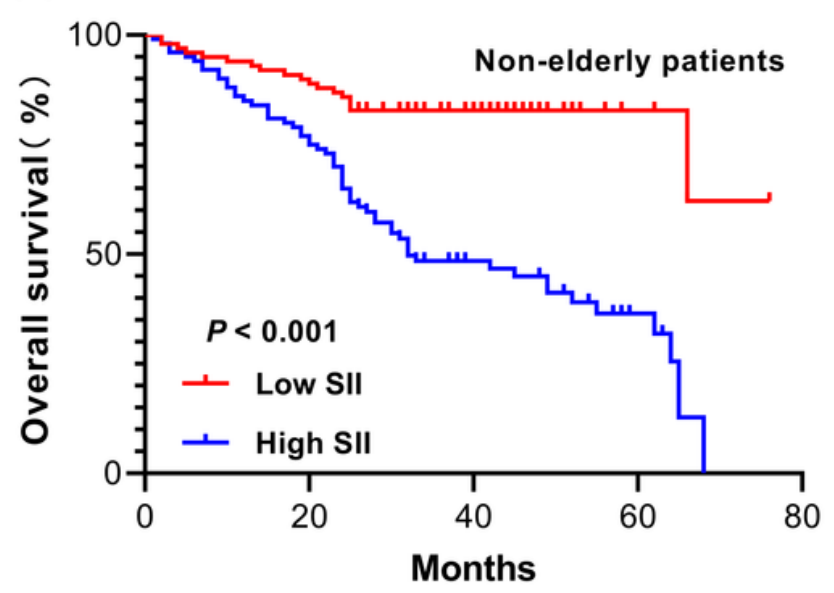

(b)

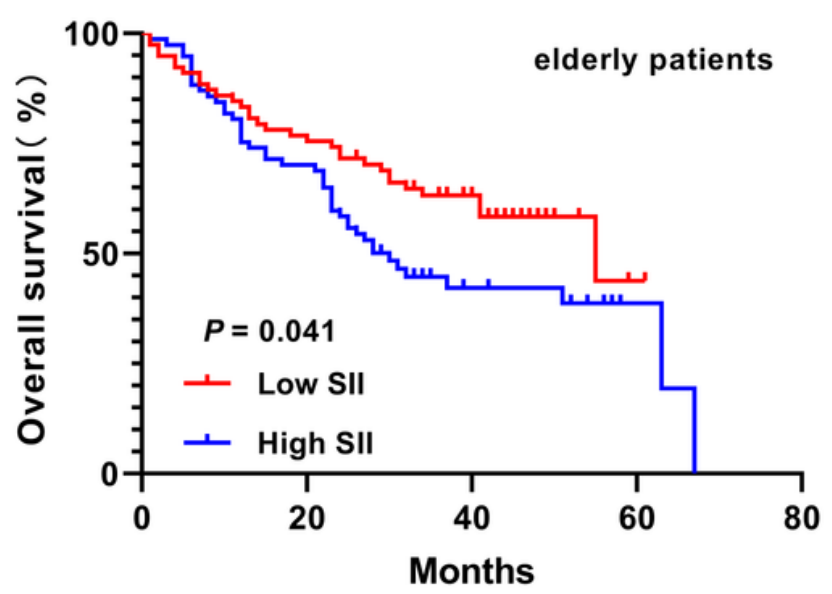

\section{Figure 5}

Correlation between the prognosis of non-elderly and elderly patients and SII after PSM. a: The 5-year OS rate in L-SII group was significantly higher than that in H-SIl group among the non-elderly patients $(62.1 \%$ vs. $31.9 \%)(P<0.001)$. b: The 5-year OS rate in L-SIl group was significantly higher than that in H-SIl group among the elderly patients (43.8\% vs. 19.4\%) $(P=0.041)$. 